\title{
Novel Few-shots Learning Neural Network for Predicting Carbohydrate-active Enzyme (CAZyme) Affinity Towards Fructo-oligosaccharides
}

Shaoxun Liu

University of Southern California

Yi Kou ( $\nabla$ yikou@usc.edu )

University of Southern California https://orcid.org/0000-0002-7139-2043

Lin Chen

University of Southern California

Research article

Keywords: Machine learning, few-shots learning, neural network, CAZyme, sugar binding affinity, resistant sugar, fructo-oligosaccharides, Poisson process

Posted Date: October 19th, 2020

DOl: https://doi.org/10.21203/rs.3.rs-92730/v1

License: (c) (i) This work is licensed under a Creative Commons Attribution 4.0 International License. Read Full License

Version of Record: A version of this preprint was published at Journal of Computational Biology on December 1st, 2021. See the published version at https://doi.org/10.1089/cmb.2021.0091. 
Title Page

2 Novel Few-shots Learning Neural Network for Predicting Carbohydrate-active

3 Enzyme (CAZyme) Affinity Towards Fructo-oligosaccharides

4 Shaoxun $\mathrm{Liu}^{1, \mathrm{a}}$, Yi Kou ${ }^{1, \mathrm{a},}$, and Lin Chen ${ }^{\mathrm{a},{ }^{*}}$

$5 \quad{ }^{1}$ Co-first Authorship

$6 \quad{ }^{a}$ Molecular and Computational Biology, Department of Biological Sciences, University

7 of Southern California, 1050 Childs Way, Los Angeles, CA 90089, USA

$8{ }^{*}$ Correspondence should be addressed to Y.K. (yikou@usc.edu) or L.C.

9 (linchen@usc.edu)

11 Abstract

12 Background: The enzymatic activity of the microbiome toward carbohydrates in the

13 human digestive system is of enormous health significance (Zou, Y., et al., 2019; Pinard,

14 D., et al., 2015). Predicting how carbohydrates through food intake may affect the

15 distribution and balance of gut microbiota remains a major challenge. Understanding

16 the enzyme-substrate specificity relationship of the carbohydrate-active enzyme

17 (CAZyme) encoded by the vast gut microbiome will be an important step to address

18 this question. In this study, we seek to establish an in-silico approach to studying the

19 enzyme-substrate binding interaction.

20 Results: We focused on the key carbohydrate-active enzyme (CAZyme) and

21 established a novel Poisson noise-based few-shots learning neural network (pFSLNN) 
22 for predicting the binding affinity of indigestible carbohydrates. This approach

23 achieved higher accuracy than other classic FSLNNs, and we have also formulated new

24 algorithms for feature generation using only a few amino acid sequences. Sliding bin

25 regression is integrated with mRMR for feature selection.

26 Conclusion: The resulting pFSLNN is an efficient model to predict the binding affinity

27 between CAZyme and common oligosaccharides. This model can be potentially applied

28 to binding affinity prediction of other protein-ligand interactions based on limited

29 amino acid sequences.

$31 \quad$ Key words

32 Machine learning; few-shots learning; neural network; CAZyme; sugar binding affinity;

33 resistant sugar; fructo-oligosaccharides; Poisson process.

\section{Background}

36 In recent years, increased attention has been paid to the human c microbiome and its

37 health effect. Those microorganisms, mostly bacteria, inhabit the human

38 gastrointestinal tract and engage in a symbiotic relationship with their host

39 (Huttenhower, C. et al., 2012) (Conlon, M. A. and Anthony, B., 2015). The species of

40 microorganism presented in the human body varies among individuals. Up to now, over

412000 species of the human microbiome have been discovered, and this number is

42 predicted to increase as more human gut microbiome samples are collected (Almeida, 
43 A. et al., 2019). Within those identified species, 997 species are proven to have 44 statistical significance in positively influencing human health, which is named probiotic 45 microbiome.

One conserved feature of the vast microbiome is the expression of carbohydrate-active enzymes (CAZyme). CAZymes are enzymes that perform the synthesis, recognition, and degradation (digestion) of carbohydrates. CAZymes are essential for the microbiome to break down the complex carbohydrates from various food sources,

51 including plant cell walls and seaweeds (Huang, L. et al., 2018). Despite its prevalence, the human genome only expresses approximately 17 CAZymes (Bhattacharya, T. et al.,

53 2015). As such, most of the food carbohydrates that are indigestible to human enzymes

54 also referred to as dietary fibers, are digested by CAZymes encoded in the gut

55 microbiome. Human gut microbiome CAZymes are highly diverse in sequences

56 (Huang, L. et al., 2018). Remarkably, CAZymes encoded by the gut microbiome

57 shows the adaptability to the carbohydrates accessible to the host (Jan-Hendrik

58 Hehemann, et al., PNAS, 109, 19786, 2012). These observations suggest that

59 understanding the enzyme-substrate specificity relationship of CAZyme of the gut

60 microbiome may provide a way to use specific carbohydrates (prebiotics) to modulate

61 population abundance and distribution of gut microbiota to promote probiotic effect.

62 Carbohydrate-binding modules (CBM) are non-catalytic modules of CAZymes that

63 facilitate substrate binding (Boraston, A.B. et al., 2004). This study will focus on 
substrate binding by CBMs of CAZymes expressed by human microbiomes.

66 Oligosaccharides, consisting of 3-10 monosaccharides, are complex carbohydrates

67 found in a wide variety of biological systems. Oligosaccharides are abundant in 68 glycolipids and glycol proteins, where they play indispensable roles in cell recognition 69 and cell adhesion. One of the commonly seen oligosaccharides is fructo70 oligosaccharide (also called oligofructan), which is mainly found in fruits and 71 vegetables and has prebiotic activity as dietary fiber. Fructans can react with reactive 72 oxygen species, and this antioxidant activity can reduce potential inflammation 73 (Franco-Robles, E. and Mercedes G. L., 2015). While being a major component of the 74 human diet, Fructo-oligosaccharides cannot be digested by native human digestive 75 enzymes (Moise, A. and Maria R., 2017). They are solely digested by the human gut 76 microbiome by bacterial CAZymes (Franco-Robles, E. and Mercedes G. L., 2015). 77 However, the detailed enzyme-substrate recognition mechanisms between these 78 prebiotic oligosaccharides and microbiome encoded CAZymes remain largely 79 unexplored. To address this question, we seek to establish a high throughput and robust 80 computational approach that can be used to predict the carbohydrate substrate 81 preference by a given CAZyme of a specific bacterial species in the human gut 82 microbiome. As a first step toward this goal, here we studied the binding of four 83 model carbohydrate substrates to the active sites of CAZyme: 1-kestose, raffinose, 84 nystose, and stachyose. These are four fructo-oligosaccharides that are shown to be 
85 digestible by the human microbiome (Hayakawa, K. et al., 1990), thus are used as 86 CAZyme binding substrates during protein docking to analyze their binding pattern. 87 These analyses will provide a structural basis for future exploration of the enzyme88 substrate specificity relationship of CAZymes in the human gut microbiome at the level 89 of big data.

91 To facilitate binding affinity prediction, we used protein models generated by I-Tasser and binding affinity predicted by Molegro Virtual Docker (MVD). Structure simulation and modeling by I-Tasser are carried out to predict the tertiary structure of a given amino acid sequence (AAS). I-Tasser is by far one of the most accurate protein structure prediction servers (MacCarthy, E. and Derrick, P., 2019) with more than 90\% quality prediction accuracy and $85.1 \%$ accuracy in assigned molecular functions (Roy, A. et al., 2010). MVD is the software used for substrate-enzyme binding predictions between selected CBMs and the four aforementioned oligosaccharides. This software is also used in the docking analysis between chlorogenic acid and aldose reductase since it

100 provides a consistent and relatively accurate score for binding models with different

101 binding energy (Naeem, S. et al., 2013). MolDock algorithm used by this software 102 provides protein cavity and substrate binding location predictions with around $87 \%$ 103 accuracy and position deviation within $2 \AA$ (Thomsen, R. and Mikael H. Christensen., 104 2006). MVD provides possible binding locations, binding energy scoring (rerank score), 105 as well as cavity related fragment sequences and substrate binding residuals. MVD is 
106 by far the most optimum docking software considering accuracy, information output,

107 and runtime.

108

109 The simulation of binding interactions between the CAZyme CBM and substrate

110 oligosaccharide is relatively accurate but highly time-consuming. An average docking

111 process for one CAZyme on the I-Tasser server is approximately 30 hours. The advent

112 of machine learning provides an efficient approach for this time cost issue. However,

113 the difficulty for these enzymes and substrates to be in full data set simulation using

114 any machine learning models is its lack of ample sample space. Heavy data training is,

115 therefore, often impossible due to the scarcity of available enzyme/substrate structures.

116 To overcome this limitation, we sought to apply few-shots learning (FSL) ideas and

117 develop our version of protein sequence-based Poisson augmentation few-shots

118 learning network.

119

120 This study aims to establish a method of predicting enzyme-substrate (protein-ligand)

121 binding affinity across an unlimited number of proteins in a given sample ensemble,

122 based on a small sample $(\sim 50)$ of enzyme-substrate docking results. Few-shot learning

123 is used to generate a neural network that is capable of differentiating the distinctive

124 classes under various goals, for example, classifying pictures of different animal species

125 will small data set (Richard, Z. et al., 2017) (Li, Z. et al., 2017). This property is

126 especially important since the high variability of AAS in proteins allows testing samples 
to consist of rather various sequences from training samples. A neural network

128 optimization algorithm of finding the loss of each round of neural network generation

129 is a core feature of few-shot learning (Garcia, V. and Joan B., 2017). The loss algorithm

130 applied is based on a prototypical neural network with adjustment of using accuracy

131 rate instead of Euclidean distance; since among the various neural networks available,

132 a prototypical neural network is the most reliable means of approach in this situation

133 thanks to its outstanding performance in the small sample space in practices (Pan, Y. et

134 al., 2019), which often outputs the prediction accuracy that has surpassed human

135 recognition (He, K. et al., 2016). By integrating with few-shot learning algorithms, the

136 prototypical neural network achieved an approximated 70\% accuracy in 5-way 5-shot

137 image classification (Richard, Z. et al., 2017).

138

139 In this study, the whole set CAZyme CBMs of probiotic human microbiomes are

140 obtained from CAZy-database (Lombard, V. et al., 2014). The over 4000 proteins are

141 clustered based on K-nearest neighbors according to the primary structure. This study

142 provides the novel idea of selecting anchor protein as bases for feature generation,

143 including cavity site and protein binding site similarity calculated through fuzzy search

144 according to anchor protein binding site fragment sequences.

146 Aim to establish an improved few-shots learning model, we bring in the data 147 augmentation through Poisson noise, since it represents the distribution of amino acid 
148 in 1D. Previous research shows that the site substitution mutation of proteins can be

149 described by the Poisson-correction method (Sadygov, R. G., 2018). Especially when

150 the substitution rate is independent among sites, Poisson -correction can best describe

151 the scenario (Grishin, N. V., 1995). In this study, since the site-dependence of

152 substitution is unknown, site-independent substitution will be assumed. In addition, we

153 mapped the data into several higher dimensions. We have also compared the Poisson

154 data augmentation with Gaussian, random, and salt-and-pepper noises.

155

156 The major significance of this study is several folds: firstly, this study takes the first

157 step towards understanding enzymatic function at the scale of the gut microbiome,

158 which is a timely topic attracting much attention. Secondly, the study establishes a

159 generalized method pipeline for future similar few shots learning in biology and is the

160 first to try FSL and noise augmentation on proteins. Since the enzyme-substrate binding

161 predictions are based on primary structure instead of the tertiary structure used in most

162 other studies, the time of protein simulation can be reduced. Thirdly, the study sets up

163 the first example for future studies of protein-substrate interactions to be performed

164 with minimal data input and limited computational power with reasonable accuracy.

165

166 Results

167

1 Prototypical neural network for Few-shot learning 
170 This experiment aims to provide a method of prediction of protein-ligand interaction

171 based on a small amount of labeled data, since acquiring the labeled training set using

172 I-Tasser and Molgro is the most time-consuming.

174 Using a small training set, though timelier, provides less accurate prediction results

175 using traditional machine learning algorithms. To achieve better performance, we

176 adopted and modified the prototypical neural network for few-shot learning. This model

177 applies to our data set in two aspects. First is that most features of the data set resemble

178 distance from a specific anchor data, which renders each data point inherent distance to

179 a calculated prototype. This feature generating technique inherently implies the protein

180 evolution tree, where proteins with similar functions from similar organisms closely

181 resemble ligand binding site structure with each other. Second is that since the training

182 set is small, multiple epochs of neural network formation are best to run to exploit the

183 random selection of the starting point of linear regression so that the neural network

184 which has the best performance during cross-validation can be selected.

186 Applying prototypical neural network algorithms increases the F1 score of prediction

187 for $18 \%$, comparing the next best machine learning model SVM (table 1). Data

188 augmentation techniques are also applied to the data set. Such a technique has been

189 used in image recognition in previous studies of artificial intelligence industries. 
190 Previous studies on using data augmentation in FSL incorporated gaussian noise and

191 linear transformations, and the prediction accuracy on EMNIST data and Face

192 recognition reached accuracies of $80.25 \%$ and $58.46 \%$ using 25 samples (Antoniou, A.

193 et al., 2017). Poisson noise augmentation was applied to the data set, and in optimum

194 configurations, it increased the F1 score of prediction by $8.67 \%$. The justification and

195 discussion were stated in the following section (table 1).

196

2 Effect of Poisson augmentation

198

199 The augmenting input sequence increases the sample size, which better supports the neural net formation when experimental data scare. The natural mutation of AAS

201 sequences is independent between each site of amino acid. The mutation rate of each

202 AA remains constant, disregarding the sequence. Assuming the probability of 203 occurrence of a specific event in a small interval of a sequence is equal to the 204 macroscopic intensity, such mutation rate can be described by a Poisson distribution, 205 where the value of lambda represents the mutation rate. This mutation rate consists of 206 both total mutation rate, the possibility that an amino acid site will mutate versus will 207 not mutate, and the amino acid-specific mutation rate, the probability of which amino 208 acid the site will mutate. The amino acid-specific mutation state was generated by 209 summarizing the occurrence frequency of each amino acid in the entire sample set. This 210 likely represents the relative abundance of each amino acid. Such a method was 
211 compared with an evenly distributed model, in which the former model has better

212 prediction results. A range of different total mutation rates was tested, and $10 \%$ gave

213 the best result. The increase of the total mutation rate exhibits a possible trade-off

214 between overfitting and information perseverance.

2163 Feature importance

218 Minimum Redundancy Maximum Relevance (mRMR) algorithm was used to calculate

219 the effectiveness and redundancy of the features. The mRMR score of each feature was

220 calculated, and the features were rearranged accordingly. Without loss of generality, a

221 sliding bin of 10-feature was used to slide over the rearranged features. Features in the

222 bin were the only input features for the FSL algorithm. F1 scores of those trails were 223 obtained and shown in figure 2.

225 Features ranking 1-40 shows a decreasing trend as expected. A second peak appeared 226 at feature group 51-60, suggesting that there are features, though redundant, are capable 227 of providing more substantial information. The redundancy of features can be explained 228 by either caused by combinations between features or the underlying scaling 229 mechanism of the mRMR algorithm. The features were further rearranged according to 230 the resulting F1 values to validate the claim above. Not to lose generality, a sliding bin 231 of size 20 was used to generate three groups, with 1-10 and 51-60 in group 1, 11-20 and 
$23241-50$ in group 2, and 21-30 and 61-70 in group 3. The resulting F1 values are shown 233 in figure 2.

236 scores. This can be explained by the possible redundancy of Sugar Binding Alignment 237 as they are basically, as mentioned in the method section, Cavity Fragment Alignment 238 with a sugar-binding coefficient matrix applied to the AA exchange matrix. This 239 suggests that the modification of the sugar-binding coefficient does not have an 240 apparent effect on the prediction ability of cavity fragments.

242 The decreasing trend shown supports the hypothesis. This suggests that the most

243 important features in this pNN neural network come from group 1 of the rearranged

244 features. Note that the F1 score of intervals 1-10 and group 1 both exceeds the F1 score 245 of all features suggesting that contradicting features downplays the predicting 246 capability of the model.

248 Table 2 listed out the types of features that are selected from the 71-feature to the top 24920 features generating the highest F1 value. High percentages of $\alpha$-Helix prediction 250 score and Cavity Fragment Alignment score was selected. The identified important 251 features retain intrinsic structural significance. $\alpha$-Helix prediction score consists of 252 individual residue count (SSSH_01) and long strand count (SSSH_02). A higher score 
253 of either feature indicates the more abundant in $\alpha$-helix (figure $4 a, 4 b$ ). Cavity

254 alignment and Sugar binding alignment scores show the possibility of occurrence of a

255 similar cavity pattern between the sample protein and a given anchor protein (figure $4 f$ ).

256 The whole sequence of sugar-binding score shows gives the average affinity of residues

257 in the sample protein to a specific ligand (figure $4 \mathrm{~g}$ ).

259 Protein samples with a high correlation in values of important features are likely to 260 preserve similar structural identities and functionality. For example, P19 is a sample 261 protein that has resulted in a perfect score aligning with anchor protein P5 (CFAL_05, 262 SBAK_05), and such a relationship is validated by AAS alignment using super 263 algorithm resulting in an RMSD of 0.389 (figure 4e). Whole sequence alignment shows 264 the level of similarity in AAS between a sample and an anchor protein. P19, though 265 possesses a drastically different sequence compared with P5, has a relatively high whole 266 sequence alignment score (WSAL_05). Thus, a high structural similarity between P5 267 and P19 is identified (figure 4c, 4d). Subsequent research indicates that P5 and P19 are 268 glycogen debranching proteins in B. glumae and A. veronii (Lim, JaeYun, et al., 2009; 269 Yang, Honghui, et al., 1996). Since B. glumae and A. veronii are of different classes, it 270 is highly possible that P5 and P19 are enzymatic proteins that underwent convergent 271 evolution. The discovery of the Glxg proteins provides evidence that this pFSLNN has 272 the potential to identify proteins of similar function regardless of phylogenic origin. 273 Using the same important features for assessment, we also identified P10 and P52 to 
274 contain similar residues in their binding cavity (figure 5). Those two proteins also come

275 from different bacteria species. Despite having drastically different AAS, the two

276 proteins showed similar key residues at their binding cavities. Such ability of this model

277 can serve to accomplish the goal of classifying the human microbiome basing on 278 enzymatic functionalities instead of 16s RNA, and be beneficial to the research of the 279 functionalities of probiotics.

\section{Discussion}

This study focuses on the novel FSL with Poisson augmentation on data sets. This idea can be used in other fields such as genomic prediction, where datasets are few. The FSL model was built upon techniques in the generation of feature matrices, which can be applied to prediction models on interactions with unknown causal features but has 287 symbolic labeled subjects as anchors. Another important finding is that certain features, 288 including cavity fragment similarity and $\alpha$-helix pattern, are important for the prediction 289 of binding affinity for resistant sugars. Moreover, the method of evaluating features by 290 sliding bin regression can be applied to other FSL learning models.

292 It should be noted that there are certain limitations for this study: a) Although it 293 facilitates the acquirement of virtual calculated binding affinity data when the dataset 294 is small and especially when the mass structure simulation is not an option, the 
295 method and traditional ways in FSL needs to be further explored for the sake of

301 prediction confidence and power. d) There are certain other FSL modes and patterns

302 which could further enhance the prediction score if Poisson augmentation is added.

303 Other methods using deep learning networks (Thapa, N. et al., 2020) may achieve better

304 results for this kind of study, but in terms of time cost and coding vector embedding, it

305 may not be very well suited to FSL framework, especially with pNN, but is definitely

306 worthy of future investigation. e) Specifically, for CAZymes, more structural features

307 involving side chain interactions with certain sugar structure types can be further

308 explored. Depending on the interacting group characteristics, evaluation scores can be

309 reranked towards certain preferences such as H-bond/aromatic stacking, and the results

310 can be regionally optimized and cross-validated globally (McCartney, L. et al., 2004).

311 Still, this study is only a small step towards understanding the CAZyme features among

312 thousands of probiotic types. The intriguing world of probiotic bacteria and their

313 CAZyme relationships, together with the charming world of FSL modeling, is definitely

314 worth substantial future works to be devoted to. 


\section{Conclusion}

317 The study focused on the binding of 4 typical resistant sugars with key carbohydrate-

318 active enzymes (CAZyme) and established a novel Poisson noise-based few-shots

319 learning neural network ( $\mathrm{PFSLNN}$ ) for predicting the binding affinity of indigestible

320 carbohydrates. This approach achieved higher F1 scores than other classic

321 FSLNNs using Poisson noise augmentation, which has never been applied in the FSL

322 fields before. The Poisson augmentation is found to be optimal at a $10 \%$ noise level.

323 During the pFSLNN establishment, we have also formulated several new algorithms

324 for generating feature matrix depending on a few linear amino acid sequences, such as

325 sliding window fuzzy search and two-dimension threshold optimization. We have also

326 evaluated feature importance by novel sliding window method. Several discoveries

327 concerning the binding pattern of the resistant sugars have been made during the

328 pFSLNN prediction: 1) Different proteins share relatively similar binding cavities and

329 patterns concerning the same sugar substrate, with the same interaction residues and 3D

330 structures around the sugar. 2) The overall structures can be quite similar even across

331 different $16 \mathrm{~S}$ classes with vastly distinctive sequences, which suggests that some key

332 residues and fragment parts far from the cavity are enough to reestablish the similar

333 same binding mode and the whole protein structures. These results suggest a new

334 binding function-based relationship between CAZymes and resistant sugars from the

335 structure perspective endowed by pFSLNN prediction. 


\section{Data collection}

339

1.1 Sample preparation

340 A list of probiotic human microbiomes that contains 997 species was adopted from

341 previous research (Forster, Samuel C., et al., 2016)). Each species was searched on the

342 CAZy database (Alisdair B. et al., 2004) for its expressed CAZymes, and all CBM's

343 AAS were downloaded. This pool of CBM contains 3749 molecules. To acquire AAS

344 with distinct features, the pool of AAS was first grouped using K-mean cluster analysis.

345 Without loss of generality, $\mathrm{h}=500$ was selected as the cutoff line, and 10 groups were

346 generated. 6 AAS were randomly chosen from each group to make up the sample set of

34760 AAS. Random selection after clustering can ensure AAS with different general

348 characteristics is evenly represented in the sample set.

350 The 60 AAS samples were uploaded to the I-Tasser server (https://zhanglab.ccmb.

351 med.umich.edu/I-TASSER/) for protein structure modeling and simulation. Substrate

352 oligosaccharide models of 1-kestose (440080), raffinose (439242), nystose (166775),

353 and stachyose (439531) were obtained from PubChem databank (Berman, H.M. et al.,

354 2000). Molgro Virtual Docker was used to detect carbohydrate-binding cavities and

355 protein-ligand binding positions. The cavity was searched for each protein, and the

356 binding position search was performed within a $15 \AA$ radius around the center of the

357 cavity after considering the sugar substrate sizes in this study. 10 binding simulations 
were performed for each protein-ligand pair. The binding position with the lowest

Rerank score was recorded. For each AAS-oligosaccharide pair, ones with Rerank score

360 below -100 were labeled as 1 , representing binding, and others were labeled as 0 ,

361 representing non-binding. Each AAS thus has four labels.

\subsection{Anchor AAS selection}

364 Assuming each AAS in different groups is distinct, one AAS from each group (10 in 365 total) were selected as anchor AAS. Those AAS were not used as testing samples in the 366 following few-shot learning process. For those 10 AAS, residues that are within $6 \AA$

367 (Biro, J. C., 2006) of the cavity site were recorded as cavity related fragments with 368 connected residues in the same fragment. Fragments of less than three residues were 369 neglected. Sugar-binding fragments were also recorded based on the binding position 370 of each oligosaccharide. Those fragments were searched for in each AAS.

372 The key concept of the feature generation pipeline is to obtain the binding pattern of 10

373 anchor AAS. The higher similarity in secondary structure, cavity fragments, and sugar-

374 binding fragments between a tested AAS and an anchor AAS suggest a higher 375 possibility for the two proteins to have the same protein-ligand binding pattern. Anchor 376 AAS always has the maximum available score when compared to its secondary 377 structure, cavity fragment, and sugar-binding fragments; thus, they were taken as 378 feature standards by the prototypical neural network and remained in training set for 
each round of learning.

1.3 Feature value matrices preparation

382 According to the secondary structure sequence returned from I-Tasser, the frequency of each AA appearing as each general secondary structure type (Helix, Sheet, Coil) was recorded. These data were used to predict secondary structure.

The AA exchange matrix was adopted from Lev Y. Yampolsky and Arlin Stoltzfus's research on The Exchangeability of Amino Acids in Proteins (Yampolsky, L.Y. and

388 Arlin S., 2005), this matrix was used to assign similarity scores when performing fuzzy search between the cavity fragments and AAS. The sugar-binding coefficient was then applied to the AA exchange matrix to generate a sugar-binding exchange matrix.

\section{Neural network Preparation}

2.1 Feature generation pipeline

394 For each AAS sample, a total of 71 features (6 from secondary structure score, 10 from

395 binding cavity alignment, 10 from whole sequence alignment, 40 sugar-binding

396 fragment alignment, 4 from sugar-binding whole sequence alignment, 1 from sample

397 AAS length) were generated according to the AAS and the matrixes mentioned above:

3986 features were generated for secondary structure score, including the estimated number

399 of promoting AA and estimated number of long consecutive representing each of the 
400 three general secondary structure types. Those parameters of secondary structure give 401 hints to the overall shape of the protein, as more helix promoting AA with less helix 402 strand suggests helix strands being longer towards a rod shape. 10 features were 403 generated from cavity fragment alignment. Fuzzy search algorithm (Algorithm 2) was 404 applied to cavity fragments generated from anchor proteins on each sample AAS to 405 search for the longest succeeding fragment chain. A higher score against either anchor 406 protein implies a higher possibility for a similar cavity to form. 10 features of the whole 407 sequence alignment score implied the possibility of the whole sequence to present 408 similar interactions between the anchor protein and sample AAS. 40 features were 409 generated from the sugar-binding AA exchange matrix. The same algorithm was 410 applied, but the AA exchange matrix has been modified according to the frequency of 411 each AA binding with a respective oligosaccharide. 4 features of whole sequence sugar412 binding scores were generated using a fuzzy search algorithm with an interaction 413 coefficient modification to the AA exchange matrix. 1 feature of AAS length was added.

415 Using fragment and whole sequence similarity as a feature instead of direct and simple 416 AAS has three advantages. Firstly, the median length of sample AAS is approximately 417 530, introducing 530 features in building a neural network is unrealistically time418 consuming. Secondly, the properties of AA cannot be linearly represented due to 3D 419 intramolecular structures. This means that the feature matrix for each AA would be 420 indefinite and hard to be quantified in only 1 dimension. Thirdly, since the sample AAS 
421 has a different length, a convolutional neural network that was to be applied would be

422 increasing its time cost. Since the aim of this study is to complete mass prediction in 423 the shortest time with only a limited sample size, applying a fixed number of features 424 that describe binding patterns would be optimum.

2.2 prototypical Neural Network $(\mathrm{pNN})$ formation

427 For the 60 sample AAS, each AAS was 1:10 augmented by Poisson noise (detailed 428 description is in the data augmentation section below). A matrix of 660 AAS samples, 429 each with 71 features and 4 labels, was generated after the feature generation pipeline. 430 AAS samples augmented from the same AAS sample, including the original AAS, were 431 defined to possess the same root. The set of AAS samples was denoted D. The set of 432110 anchor AAS were denoted $\mathrm{D}^{\text {anchor }}$, where $\mathrm{D}^{\text {anchor }} \in \mathrm{D}$. 110 samples of 10 different 433 roots from $\mathrm{D}-\mathrm{D}^{\text {anchor }}$ was randomly selected as the training set, denoted as $\mathrm{D}^{\text {train }}$, the 434 remaining is the testing set, denoted $\mathrm{D}^{\text {test }}$. The ratio between the training and testing set 435 is $5: 1$.

437 For each epoch of FSL training, $\mathrm{D}^{\text {train }}$ was divided into supporting set $\mathrm{S}$ and query set 438 Q, where the number of samples in $\mathrm{S}$ and the number of samples in Q has a ratio of 4:1. 439440 AAS samples of 40 different roots, including $\mathrm{D}^{\text {Anchor }}$ were used to compute the 440 prototype from S. The training algorithm of the Few-Shot learning model is the same 441 as Jake Snell, Kevin Swersky, and Richard Zemel's (Richard, Z. et al., 2017). D ${ }^{\text {Anchor }}$ 
442 was always included in the supporting set as they provided the guidelines of each

443 feature. Anchor proteins contributed the most to the class generation as they have the 444 most distinct feature values. The neural network generated that performs the 445 classification is denoted NN. Q, consisting of the leftover $110 \mathrm{AAS}$, is used to compute 446 Loss-J of NN. Loss-J was modified to be the number of incorrect predictions of this 447 neural network on the validation set. As epochs proceed to 100, the NN with the least 448 loss-J was selected to be returned as the best neural network (bNN). The prediction 449 accuracy of bNN for $\mathrm{D}^{\text {test }}$ was recorded.

\section{Feature generation}

$452 \quad 3.1$ Generation of secondary structure score

453 -Feature structure

454 Each amino acid sequence (AAS) input returned 6 values that consist of a secondary 455 structure score, denoted as $\operatorname{Mij}(i \in[1,3], i \in[1,2])$. Those values included estimation 456 in the number of Amino acids (AA) promoting each classic secondary structure 457 (Helixes, plated Sheets, and random Coils) that were denoted as H, S, and C, as well as 458 the estimation of the number of long, consecutive strands $(\geq 5)$ of AA promoting the 459 same secondary structure. To be convenient as a demo, we defined a fragment of AAS, 460 showing the consecutive occurrence of the same classic secondary structure over 5 461 times as a secondary structure strand. 
464 the secondary structure promotion matrix ( $\mathrm{sspM}$ ) and secondary structure promotion 465 threshold matrix (sspT). sspM recorded the frequency of each AA appearing in each 466 classic secondary structure after normalization according to the average and standard 467 deviation of the training set. The normalization of this matrix allowed the values to be 468 in a statistical range. sspT contained three sets of thresholds, which are the promotion 469 bar, demotion bar, and the tolerance number. The two bars characterized AA into 470 Promoting, indifferent, and demoting for each of the three secondary structure types.

471 The promotion bar is the lower bound of the sspM value of a given AA being 472 characterized to start or succeed a secondary structure strand. The demotion bar is the 473 higher bound of the sspM value of a given AA being characterized to prohibit or 474 terminate a secondary structure strand. AA with sspM value between the two thresholds 475 is considered indifferent to a classic secondary structure type. The tolerance number is 476 the minimum number of AA in a secondary structure strand to view the next indifferent 477 AA as a successor of the ongoing secondary structure strand.

480 The data input that generates those matrixes consisted the AAS training set, denoted $481 A^{\text {trian }}{ }_{i j}\left(i \in[1,50], j \in\left[1\right.\right.$, length $\left.\left.\left(A^{\operatorname{trian}_{i}}\right)\right]\right)$, where $i$ and $j$ mean the $j^{\text {th }} A A$ from the $i^{\text {th }}$ AAS 482 of the set, and the secondary structure sequence (SSS) returned by I-Tasser, denoted 483 $\mathrm{S}^{\operatorname{trian}_{\mathrm{ij}}}\left(\mathrm{i} \in[1,50], \mathrm{j} \in\left[1\right.\right.$, length $\left.\left.\left(\mathrm{S}_{\mathrm{i}}^{\mathrm{trian}}\right)\right]\right)$. 
485 The data collection for $\mathrm{m}_{\mathrm{xy}} \in \operatorname{sspM}(\mathrm{x} \in[1,20], \mathrm{y} \in[1,3])$, where $\mathrm{m}_{\mathrm{xy}}$ represents the $\mathrm{x}^{\text {th }}$ 486 AA, or the AA " $\mathrm{x}$ ", and the $\mathrm{y}^{\text {th }}$ classic secondary structure, is shown below. $\mathrm{m}_{\mathrm{xy}}$ finds 487 the portion between an AA in the helix (sheet, coil) and the total amount of the AA type, 488 normalized by the average and standard deviation of all three classic secondary 489 structure types.

$490 \quad m_{x y}$

$491=\frac{-\overline{m_{y}}+\frac{\sum_{i, j \in\left(A_{i j}^{\text {Train }}=x\right)} f\left(A_{i j}^{\text {Train }}\right)}{\sum_{i, j \in\left(A_{i j}^{\text {Train }}=x\right)} 1}}{\sigma_{m_{y}}}$

$492 f\left(A_{i j}^{\text {Train }}\right)$

$493=\left\{\begin{array}{l}1, A_{i j}^{\text {Train }}=S_{i j}^{\text {Train }} \\ 0, A_{i j}^{\text {Train }} \neq S_{i j}^{\text {Train }}\end{array}\right.$

494 The threshold matrix $t_{x y} \in \operatorname{sspT}(x \in[1,3], y \in[1,3])$, where $t_{x y}$ represents the $x=1$ 495 (higher), $x=2$ (lower), $x=3$ (tolerance) threshold for the $\mathrm{y}^{\text {th }}$ classic secondary structure, 496 was optimized through linear regression of minimizing difference between GenSS() 497 output value of $\mathrm{A}^{\text {trian }}{ }_{\mathrm{ij}}$ and $\mathrm{S}^{\text {trian }}{ }_{\mathrm{ij}}$. A demo run of GenSS() using the values from a given 498 sspT was denoted by $\operatorname{GenSS}^{t}()$. The sspT was optimized when the difference between 499 the estimated value and real value (running SSS in the same algorithms gives the real 500 value) is minimized.

501 $\frac{d \sum \frac{\mid \text { GenSS }^{t}\left(A^{\text {train }}\right)-\operatorname{GenSS}^{t}\left(S^{\text {train }}\right) \mid}{\operatorname{GenSS}^{t}\left(S^{\text {train }}\right)}}{d t}$ $502=0$ 
503 GenSS() has two parts: the first is counting the number of each secondary structure

504 promoting AA. For the $\mathrm{c}^{\text {th }}$ AAS, there are:

$505 \quad M_{i 1}$

$506=\sum_{j \in A_{c j}^{\text {Train }}} f\left(A_{c j}^{\text {Train }}\right)$

$507 f\left(A_{c j}^{\text {Train }}\right)$

$508=\left\{\begin{array}{l}1, m_{A_{c j}^{\text {Train }}}{ }_{i} \geq \mathrm{t}_{1 i} \\ 0, m_{A_{c j}^{\text {Train }}}<\mathrm{t}_{1 i}\end{array}\right.$

509 The generation of $\mathrm{M}_{\mathrm{i} 2}$ is further illustrated in Figure 8 .

$511 \quad 3.2$ Generation of fragment binding and whole sequence binding score

512 -AA exchange fuzzy search

513 Fuzzy search is a searching algorithm based on the sliding-window idea with a penalty

514 of the difference applied to each distinctive element in the window (Vernica, R. and

515 Chen L., 2009). The advantage of the sliding-window algorithm against the Smith-

516 Waterman algorithm is that the Smith-Waterman algorithm aims to find local

517 alignments between the two strands, which neither must include the other, while the

518 sliding-window algorithm ensures to find consecutive and including alignments. In

519 addition, the Smith-Waterman algorithm aims to find the aligning strand while the aim

520 of the fuzzy search is to return the alignment score for each site.

522 The substitution matrix in this alignment was the AA exchange matrix, denoted aaEX $\mathrm{ij}_{\mathrm{ij}}$ 
$523(\mathrm{i} \in[1,20], \mathrm{j} \in[1,20])$, where $\mathrm{i}$ is the substituting AA, and $\mathrm{j}$ is the substituted AA. The

524 score for the same AA substitution is 1000 , and the higher score indicated better

525 substitution efficiency. One AAS of sequence and fragment were inputs for one round

526 of fuzzy search. The fragment was being searched throughout the sequence. The

527 sequence is denoted $\mathrm{S}$, where $\mathrm{S}_{\mathrm{m}}$ is the $\mathrm{m}^{\text {th }} \mathrm{AA}$ of the sequence. The fragment is denoted

$528 \mathrm{~F}$, where $\mathrm{F}_{\mathrm{n}}$ is the $\mathrm{n}^{\text {th }}$ AA of the fragment. The Fuzzy search returns a vector of length

$529 \mathrm{~m}$, denoted $\mathrm{R}_{\mathrm{m}}$. Each value of $\mathrm{R}$ represented the alignment score between $\mathrm{S}$ and $\mathrm{F}$ at the

530 given position. The value was the average of aaEX values substituting each AA from

531 the sequence for the AA from the fragment.

$532 R_{i}=$

$533 \quad \frac{\sum_{j=1}^{\text {length }(F)} a a E X_{S_{i+j-1} F_{j}}}{\operatorname{length}(F)}$

535 -Longest increasing fragment

536 A vector of cavity fragments was obtained from each anchor protein. Each fragment

537 was labeled by its order in the anchor protein sequence. A new vector $\mathrm{V}$ of length $\mathrm{m}$

538 was first filled with placeholder values. For each $\mathrm{R}_{\mathrm{xm}}$, which $\mathrm{x}$ indicates the $\mathrm{x}^{\text {th }}$ fragment

539 from the anchor, looping through $\mathrm{R}_{\mathrm{x}}$, each $\mathrm{i}(\mathrm{i} \in[1, \mathrm{~m}])$ that has $\mathrm{R}_{\mathrm{xi}}$ larger than threshold

$540 \quad$ value $\mathrm{T}, \mathrm{V}_{\mathrm{i}}$ is labeled $\mathrm{x}$.

$541 \quad V_{i}=f\left(R_{x i}\right) \quad f\left(R_{x i}\right)$

$542=\left\{\begin{array}{l}0, R_{x i}<T \\ x, R_{x i} \geq T\end{array}\right.$

543 A second vector $\mathrm{W}$ of the same length as $\mathrm{V}$ stored the fuzzy search alignment value of 
544 each corresponding position. A longest increasing subsequence searching algorithm

545 (Aldous, D. and Persi D., 1999) was applied to V with the weight of each position

546 modified to its corresponding value in $\mathrm{W}$. The returning cavity fragment alignment

547 score was the total weight of the longest increasing subsequence in $\mathrm{V}$ that was divided

548 by the total number of AA in all the fragments.

550 -Two-dimensional optimization of threshold $\mathrm{T}$

551 Threshold $\mathrm{T}$ was a crucial parameter in the search for the longest cavity strand. This

552 parameter was decided to increase the standard deviation of each column vector of

553 feature matrix while maintaining the minimum difference of such standard deviation

554 across the 10 features. We denote each column vector of the 10 cavity fragment

555 alignment of all samples as $F_{i}(i \in[1,10])$, where $i$ indicates the cavity fragment

556 alignment vector with the $\mathrm{i}^{\text {th }}$ anchor protein. The standard deviation of $F_{i}$ using $t$ as

557 threshold $\mathrm{T}$ is denoted $\mathrm{sdF}_{\mathrm{i}}^{\mathrm{t}}$. The standard deviation of $\mathrm{sdF}_{\mathrm{i}}^{\mathrm{t}}$ for $\mathrm{i} \in[1,10]$, is denoted

$558 \mathrm{sd}\left(\mathrm{sdF}^{\mathrm{t}}\right) . \mathrm{t}$ that fulfills equation 8 was chosen as $\mathrm{T}$. $\mathrm{T}$ value is 608 in the experimental

559 run.

$560 \frac{d \frac{\sum_{i=1}^{10} s d F_{i}^{t}}{s d\left(s d F^{t}\right)}}{d t}$

$561=0$

563 -Whole sequence fuzzy search

564 For the whole sequence alignment score, the shorter sequence between the anchor 
566 the previous section is applied with the substituting and substituted AA assigned

567 according to the compared length between the two AA. AA was substituted from the 568 sample AAS to the anchor AAS. The fuzzy search alignment value was returned as the 569 whole sequence alignment score.

$571 \quad$-Sugar binding matrix

5724 sugar-specific binding matrix was multiplied with a coefficient to aaEX to form sugar-

573 binding AA exchange matrix denote $\operatorname{sbEX}_{m n}^{\mathrm{i}}(\mathrm{i} \in[1,4], \mathrm{m}, \mathrm{n} \in[1,20])$, where $\mathrm{i}$

574 represents 1-kestose, nystose, raffinose, and stachyose, $\mathrm{n}$, and $\mathrm{m}$ represent substituting

575 AAm with AA n. 4 vectors of AA-sugar interaction frequency, denoted $\operatorname{sbV}_{\mathrm{j}}(\mathrm{j} \in[1,20])$,

576 where $\mathrm{j}$ represents AA. For $\mathrm{i} \in[1,4]$, the AA-sugar affinity matrix was generated by

577 counting AA residuals that appear within $5 \AA$ (Sharma, R. et al., 2008) of the sugar-

578 binding site. This method is similar to the method introduced in the work by Misaki

579 Banno when performing AA-sugar affinity prediction (Banno, M. et al., 2017). sbM ${ }^{\mathrm{i}}{ }_{\mathrm{mn}}$

580 was a matrix that contains the ratio between $\mathrm{sbV}_{\mathrm{n}}$ and $\mathrm{sbV} \mathrm{m}$. A larger ratio represents a

581 higher affinity of substituted AA. sbEX ${ }^{i}$ was generated by applying a sbM $^{\mathrm{i}}$ filter, 582 multiplied by a factor $\mathrm{F}$, on aaEX. 
$584=a a E X$

$585 \times(1+F$

$586 \times\left(s b E X_{m n}^{i}\right.$

$587-1))$

588 F was also optimized using equation 8 , replacing $\mathrm{T}$ by $\mathrm{F}$.

3.3 Whole sequence sugar-binding

5914 whole sequence sugar-binding score, one for each oligosaccharide ligand, was

592 generated. AA-sugar interaction matrix was obtained using the same method as above.

593 The difference is that AA in the whole sequence was accounted for instead of AA

594 residuals that appear within $5 \AA$ of the sugar-binding site. And the average of AA-sugar

595 interaction score for all AA in the protein was calculated as the whole sequence sugar-

596 binding score.

598 3.4 Poisson augmentation

599 Poisson augmentation simulates the mutation of AAS to increase the sample size. The

600 usage of Poisson distribution relied on the assumptions 1) AA mutation chance is 601 independent of AA site; 2) the effect of minor mutations will not affect sugar-binding 602 efficiency. This augmentation process required an AA frequency matrix, denoted aaFMi $603(\mathrm{i} \in[1,20])$, where $\mathrm{i}$ represents $\mathrm{AA}$, and a mutation chance at each site $\mathrm{C}$. The 
604 probability mass function of Poisson distribution is given by function (10). The value

605 of $\mathrm{k}=1$ and the value of $\lambda$ was calculated by equation (11).

$606 p$

$607=\frac{\lambda^{k} e^{-\lambda}}{k !}$

$608 \lambda_{i}$

$609=C$

$610 \times \frac{a a F M_{i}}{\sum a a F M}$

$61120 \lambda$ values formed an accumulated interval sequence. After an AAS was input to the

612 Poisson augmentation function, a random number from 0 to 1 was generated for each

613 site of AA. The interval in which the random number falls into determines if the AA at

614 this given site would mutate and which AA it would mutate to.

615

616 The Poisson augmentation function was run on each sample AAS for 10 times to

617 generate a set of augmented AAS of the same root. Test trials of $\mathrm{C}=5 \%, 10 \%$, and $20 \%$

618 were performed, and $\mathrm{C}=10 \%$ gave the best prediction results. Augmented AAS ran the

619 same feature generation pipeline.

620

621 List of abbreviations

622 CAZyme Carbohydrate-active Enzyme

623 FSL Few-Shots Learning

624 FSLNNs Few-Shots Learning Neural Network 


$\begin{array}{lll}625 & \text { pNN } & \text { prototypical Neural Network } \\ 626 & \text { pFSLNN } & \text { Poisson noise-based Few-Shots Learning Neural Network } \\ 627 & \text { mRMR } & \text { minimum Redundancy Maximum Relevance } \\ 628 & \text { CBM } & \text { Carbohydrate-Binding Modules } \\ 629 & \text { MVD } & \text { Molegro Virtual Docker } \\ 630 & \text { AA } & \text { Amino Acid } \\ 631 & \text { AAS } & \text { Amino Acid Sequence } \\ 632 & \text { SVM } & \text { Support Vector Machines } \\ 633 & \text { SspM } & \text { secondary structure promotion Matrix } \\ 634 & \text { SspT } & \text { secondary structure promotion Threshold matrix } \\ 635 & \text { SSS } & \text { Secondary Structure Sequence } \\ 636 & \text { aaEX } & \text { amino acid EXchange matrix }\end{array}$

637

638 Declarations

639 Ethics approval and consent to participate

640 Not applicable

641

642 Consent for publication

643 Not applicable

644

645 Availability of data and materials 


\section{Competing interests}

649 The authors declare that they have no competing interests.

650

\section{Authors' contributions}

652 S. L and Y. K designed the model and implemented the study. S. L, Y. K and L. C drafted

653 the manuscript. Y. K and L. C supervised the whole project. All authors have read and 654 approved the manuscript.

655

$656 \quad$ Funding

657 No funding was obtained for this study

658

\section{Acknowledgements}

660 Great thanks to Professor Richard Zemel, Nicolas Terrapon, Alisdair Boraston, Dukka

$661 \mathrm{KC}$, and Bruce Tidor for their kind and informative discussion.

662

663 Reference

664 Aldous, D. and Persi D. (1999) Longest increasing subsequences: from patience sorting 665 to the Baik-Deift-Johansson theorem. Bulletin of the American Mathematical Society $666 \quad 36.4: 413-432$. 
667 Alisdair B. et al. (2004) Carbohydrate-binding modules: fine-tuning polysaccharide 668 recognition. Biochemical journal 382.3: 769-781.

669 Almeida, A. et al. (2019) A new genomic blueprint of the human gut microbiota. Nature $670 \quad 568.7753: 499-504$.

671 Antoniou, A. et al. (2017) Data augmentation generative adversarial networks. arXiv 672 preprint arXiv:1711.04340.

673 Berman, H.M. et al. (2000) The Protein Data Bank Nucleic Acids Research, 28: 235674242.

675 Bhattacharya, T. et al. (2015) Global profiling of carbohydrate active enzymes in human 676 gut microbiome. PloS one 10.11: e0142038.

677 Biro, J. C. (2006) Amino acid size, charge, hydropathy indices and matrices for protein 678 structure analysis. Theoretical Biology and Medical Modelling 3.1: 15.

679 Bonk, B. M. et al. (2019) Machine Learning Identifies Chemical Characteristics That 680 Promote Enzyme Catalysis. Journal of the American Chemical Society 141.9: 41086814118.

682 Banno, M. et al. (2017) Development of a sugar-binding residue prediction system from 683 protein sequences using support vector machine. Computational biology and chemistry 684 66: $36-43$.

685 Boraston, A.B. et al. (2004) Carbohydrate-binding modules: fine-tuning polysaccharide 686 recognition. Biochemical journal 382.3: 769-781.

687 Conlon, M. A. and Anthony, B. (2015) The impact of diet and lifestyle on gut 
688

690

691 acids research 44.D1: D604-D609.

692 Franco-Robles, E. and Mercedes G. L. (2015) Implication of fructans in health:

694 Garcia, V. and Joan B. (2017) Few-shot learning with graph neural networks. arXiv 695 preprint arXiv:1711.04043.

696 Grishin, N. V. (1995) Estimation of the number of amino acid substitutions per site 697 when the substitution rate varies among sites. Journal of molecular evolution 41.5: 675698679.

699 Han, X. et al. (2018) Fewrel: A large-scale supervised few-shot relation classification 700 dataset with state-of-the-art evaluation. arXiv preprint arXiv:1810.10147.

701 Hayakawa, K. et al. (1990) Effects of soybean oligosaccharides on human faecal flora. 702 Microbial Ecology in Health and Disease 3.6: 293-303.

703 He, K. et al. (2016) Deep residual learning for image recognition. Proceedings of the 704 IEEE conference on computer vision and pattern recognition. 770-778

705 Huang, L. et al. (2018) dbCAN-seq: a database of carbohydrate-active enzyme 706 (CAZyme) sequence and annotation. Nucleic acids research 46.D1: D516-D521.

707 Huttenhower, C. et al. (2012) Structure, function and diversity of the healthy human 708 microbiome. Nature 486.7402: 207. 
709 Li, Z. et al. (2017) Meta-sgd: Learning to learn quickly for few-shot learning. arXiv

710 preprint arXiv:1707.09835.

711 Lim, JaeYun, et al. (2009) Complete genome sequence of Burkholderia glumae BGR1.

712 Journal of bacteriology 191.11: 3758-3759.

713 Lombard, V. et al. (2014) The carbohydrate-active enzymes database (CAZy) in 2013.

714 Nucleic acids research 42.D1: D490-D495.

715 MacCarthy, E. and Derrick, P. (2019) Advances in protein super-secondary structure

716 prediction and application to protein structure prediction. Protein Supersecondary

717 Structures. Humana Press, New York, NY. 15-45.

718 McCartney, L. et al. (2004) Glycoside hydrolase carbohydrate-binding modules as 719 molecular probes for the analysis of plant cell wall polymers. Analytical biochemistry $720 \quad 326.1: 49-54$.

721 Moise, A. and Maria R. (2017) The Gut Microbiome: Exploring the Connection 722 Between Microbes, Diet, and Health. ABC-CLIO.

723 Montserrat, D. et al. (2017) Training object detection and recognition CNN models 724 using data augmentation. Electronic Imaging 2017.10: 27-36.

725 Naeem, S. et al. (2013) Docking studies of chlorogenic acid against aldose redutcase 726 by using molgro virtual docker software. Journal of Applied Pharmaceutical Science $727 \quad 3.1: 13$.

728 Ndeh, D. et al. (2017) Complex pectin metabolism by gut bacteria reveals novel 729 catalytic functions. Nature 544.7648: 65-70. 
730 Pan, Y. et al. (2019) Transferrable prototypical networks for unsupervised domain

731 adaptation. Proceedings of the IEEE Conference on Computer Vision and Pattern

732 Recognition. 2239-2247

733 Pinard, D., et al. (2015) "Comparative analysis of plant carbohydrate active enZymes

734 and their role in xylogenesis." BMC genomics 16.1: 1-13.

735 Richard, Z. et al. (2017) Prototypical networks for few-shot learning. Advances in

736 neural information processing systems. 4077-4087.

737 Roy, A. et al. (2010) I-TASSER: a unified platform for automated protein structure and 738 function prediction. Nature protocols 5.4: 725-738.

739 Sadygov, R. G. (2018) Poisson model to generate isotope distribution for biomolecules.

$740 \quad$ Journal of proteome research 17.1: 751-758.

741 Sharma, R. et al. (2008) The interaction of carbohydrates and amino acids with aromatic

742 systems studied by density functional and semi-empirical molecular orbital calculations

743 with dispersion corrections. Physical Chemistry Chemical Physics 10.19: 2767-2774.

744 Thapa, N. et al. (2020) DeepSuccinylSite: a deep learning based approach for protein

745 succinylation site prediction. BMC bioinformatics 21: 1-10.

746 Thomsen, R. and Mikael H. Christensen. (2006) MolDock: a new technique for high-

747 accuracy molecular docking. Journal of medicinal chemistry 49.11: 3315-3321.

748 Yang, Honghui, et al. (1996) Coordinate genetic regulation of glycogen catabolism and 749 biosynthesis in Escherichia coli via the CsrA gene product. Journal of bacteriology $750 \quad$ 178.4: 1012-1017. 
751 Vernica, R. and Chen L. (2009) Efficient top-k algorithms for fuzzy search in string 752 collections. Proceedings of the First International Workshop on Keyword Search on 753 Structured Data. 9-14.

754 Yampolsky, L.Y. and Arlin S. (2005) The exchangeability of amino acids in proteins. 755 Genetics 170.4: 1459-1472.

756 Zou, Y., et al. (2019) "1,520 reference genomes from cultivated human gut bacteria 757 enable functional microbiome analyses." Nature biotechnology 37.2: 179-185.

\section{Figure legends}

760 Figure 1: Fructo-Oligosaccharides. Name, CAS number, 3D structure, and 2D structure

761 of the four fructo-oligosaccharides that are used as substrates in this study.

762 Figure 2: F1 scores of FSL models trained using each 10-feature groups arranged 763 according to mRMR scores.

764 Figure3: F1 score of FSL models inputting 20 features from three groups of mRMR 765 intervals.

766 Figure 4: Structural presentation of sample proteins. a) Structure of P9 with alpha 767 helixes in red. SSSH_01 $=12$, SSSH_02 $=150$. b) Structure of P6 with alpha helixes 768 in red, SSSH_01 $=$ 1, SSSH_02 = 35. c) Structure of anchor protein P5 with alpha 769 helixes in blue and beta sheets in yellow; binding ligand1-kestose is shown in red. d)

770 Structure of protein P19 with alpha helixes in blue and beta sheets in yellow; binding 771 ligand1-kestose is shown in red. e) Structural alignment of cavity fragments of P5 (blue) 
772 and P19 (gray) using align function in pymol with alignment RMSD $=0.389$; residue

773 labels are shown in corresponding colors. f) Sample AAS alignment of P5 (GlgX [B.

774 glumae]) and P19 (GlgX [A. veronii]) around the two cavity fragments; aligned AA of

775 a given secondary structure or belongs to a cavity fragment is shown in the color scheme.

776 g) Sample AAS alignment of non-cavity parts of P5 and P19, with AA of a given

777 secondary structure shown in the color scheme.

778 Figure 5: Predicted interaction of two CAZyme CBMs (P10 and P52) with 1-kestose.

779 P10 and P52 are highly correlated according to group 1 features, while having no

780 significant similarity in sequence. Similar amino acid residues are found in the radius

781 of the interaction of both P10 and P52, including SER, ALA, ARG, and LEU. This

782 suggests that the feature matrix may obtain inherent biological meaning. a) Interaction

783 model of P10 viewing from beta-D-fructofuranose. b) Interaction model of P10 viewing

784 from beta-D-fructofuranosyl residue. c) Interaction model of P10 viewing from alpha-

785 D-glucopyranosyl residue. d) Interaction model of P52 viewing from beta-D-

786 fructofuranose. e) Interaction model of P52 viewing frombeta-D-fructofuranosyl

787 residue. f) Interaction model of P52 viewing from talpha-D-glucopyranosyl residue.

788 Figure 6: Flow chart for feature generation procedure. Arrows show derivation.

789 Figure 7: Flow chart of feature matrix generation and pFSLNN process.

790 Figure 8: This figure illustrates the bases of the algorithm estimating the number of

791 residuals of each general secondary structure type. Without loss of generality, $\alpha$-Helix

$792(\mathrm{H})$ is shown as an example. A cursor scans over the input AAS to find the first $\mathrm{H}$ 
793 promoting AA. A secondary structure strand of $\mathrm{H}$ begins. When the strand length is

794 more than or equal to 5, the strand is considered a long strand. As the cursor proceeds,

7953 types of successor (promoting, demoting, indifferent) AA can result in 5 cases. Initial

$796 \operatorname{sspTH}=(-0.5,-1.6,2)$.

797

798 Tables

799 Table 1:

Accuracy and F1 score for experimented models

\begin{tabular}{lccc}
\hline Method & Augmented & Accuracy & F1 score \\
\hline Random Forest & $\mathrm{N}$ & $67.70 \%$ & $44.36 \%$ \\
SVM & $\mathrm{N}$ & $72.50 \%$ & $52.13 \%$ \\
FSL unaugmented & $\mathrm{N}$ & $66.55 \%$ & $70.13 \%$ \\
FSL Poisson aug (5\%) & $\mathrm{Y}$ & $67.00 \%$ & $76.92 \%$ \\
FSL Poisson aug (10\%) & $\mathrm{Y}$ & $69.15 \%$ & $78.67 \%$ \\
FSL Poisson aug (20\%) & $\mathrm{Y}$ & $66.55 \%$ & $77.24 \%$ \\
FSL Poisson aug (30\%) & $\mathrm{Y}$ & $65.75 \%$ & $75.38 \%$ \\
FSL Poisson aug (40\%) & $\mathrm{Y}$ & $66.15 \%$ & $68.47 \%$ \\
FSL Salt \& Pepper aug (10\%) Y & $65.00 \%$ & $67.73 \%$ \\
FSL Random mutation (10\%) Y & $67.45 \%$ & $73.22 \%$ \\
FSL Gaussian aug (10\%) & $\mathrm{Y}$ & $65.50 \%$ & $65.87 \%$
\end{tabular}

800 Table 2:

Percentage of selected feature types in the top 20 features

\begin{tabular}{lccc} 
Feature Type & Total & Top 20 & Percent Selected \\
\hline Cavity Fragment Alignment & 10 & 4 & $40 \%$ \\
Whole sequence Sugar Binding & 4 & 3 & $75 \%$ \\
Whole sequence Alignment & 10 & 2 & $20 \%$ \\
$\alpha$-Helix prediction & 2 & 2 & $100 \%$ \\
Sugar binding Alignment & 40 & 9 & $22.5 \%$
\end{tabular}




\section{Figures}

\section{Resistant Oligossacharides}

1-kestose

CAS: 470-69-9
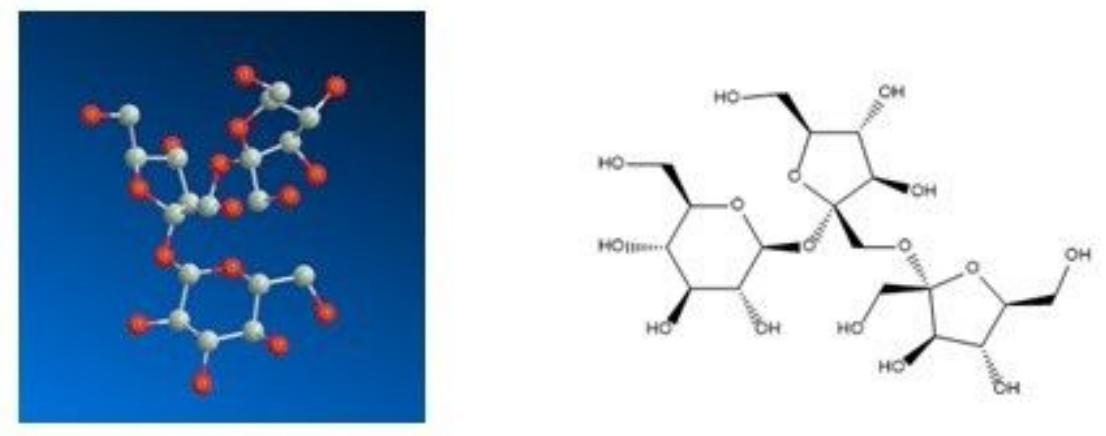

Raffinose

CAS: $512-69-6$
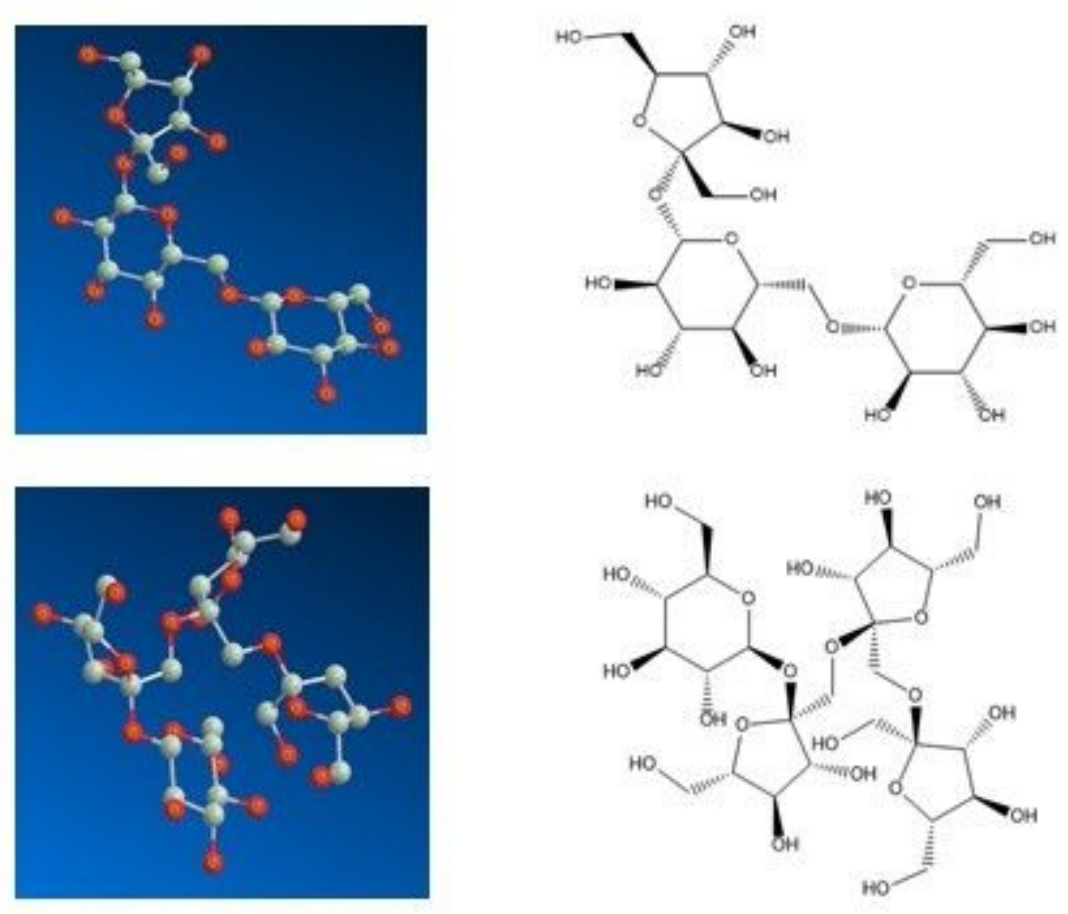

Nystose

CAS: 13133-07-8

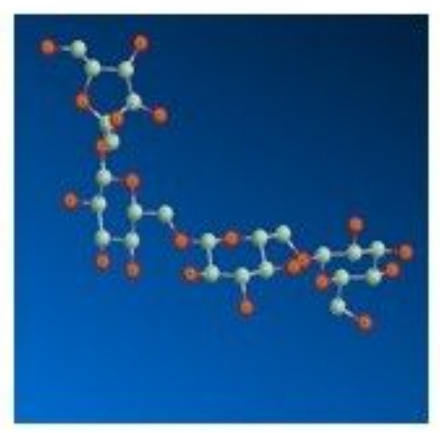

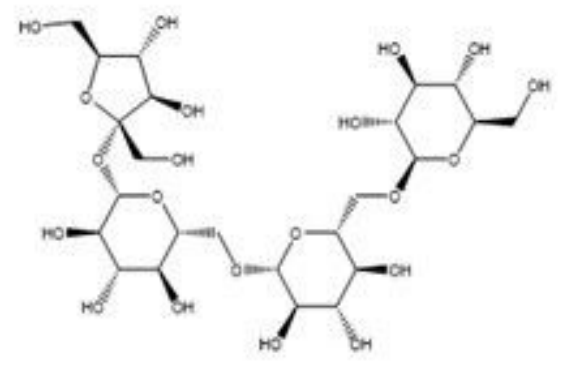

Stachyose:

CAS: 470-55-3 
F1 Score of mRMR Intervals

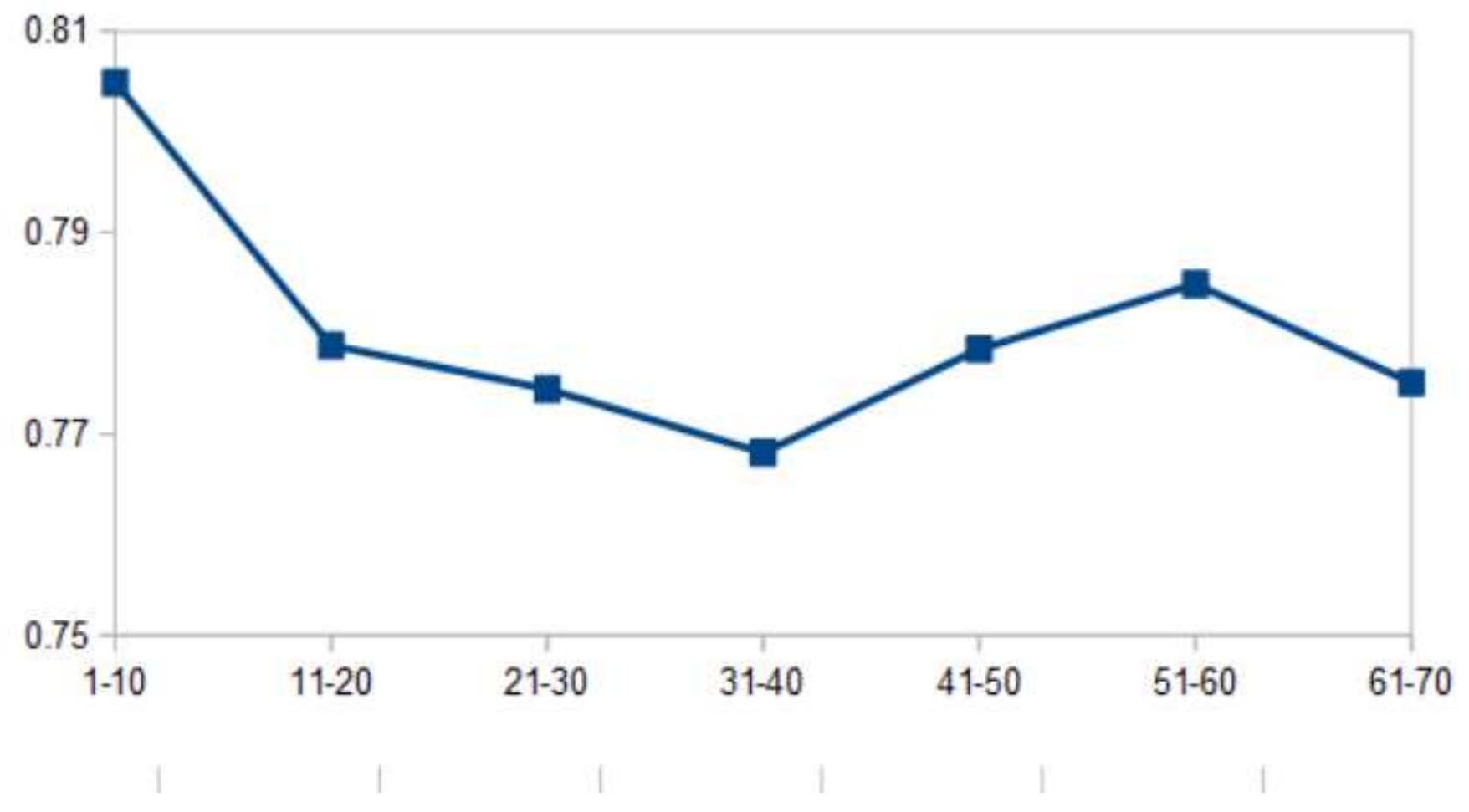

Figure 2

F1 scores of FSL models trained using each 10-feature groups arranged according to mRMR scores.

F1 Score of Rearranged Groups

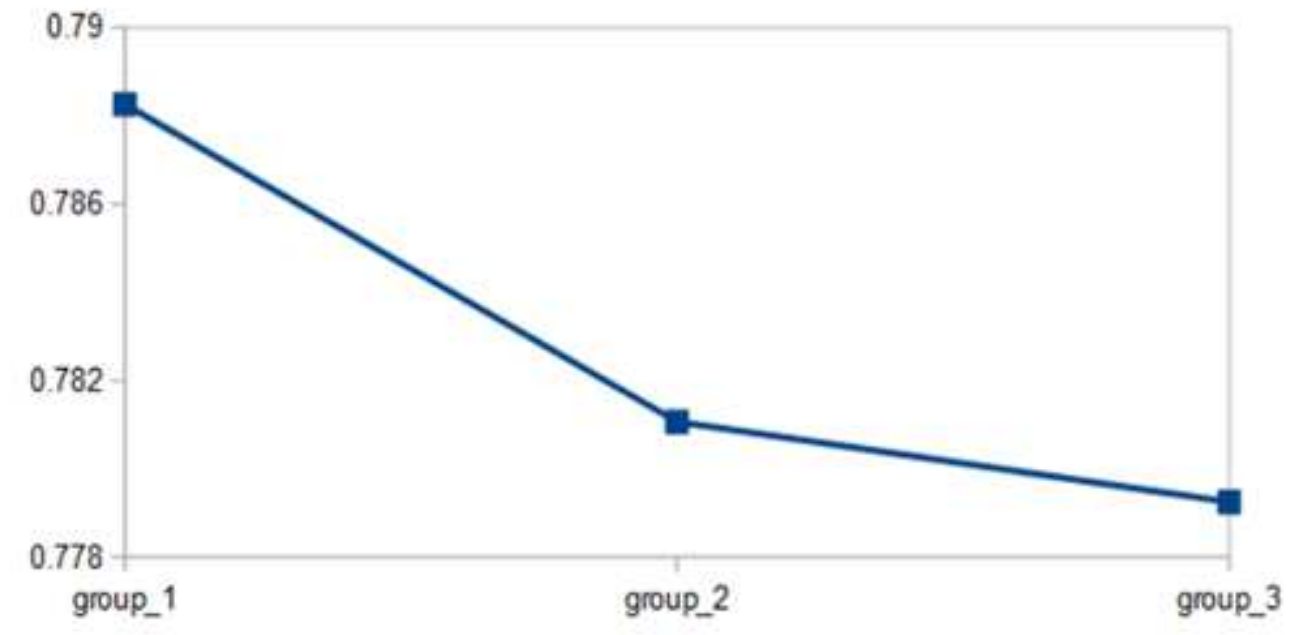

Figure 3

F1 score of FSL models inputting 20 features from three groups of mRMR intervals. 
a

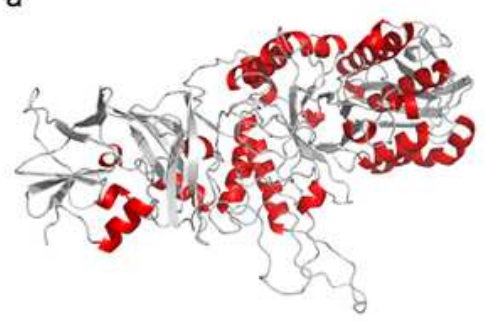

C
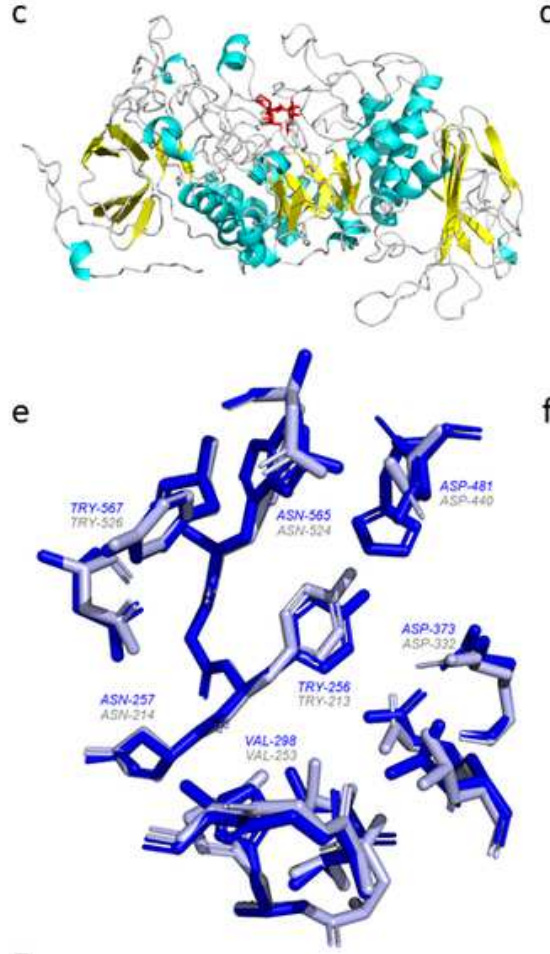

g GlgX [B. glumae BGR1]

30 IQEGLPFPLGATWGHGVNFALFAHATKVELCLFDEDGKTERERIALPEYTDEVFHVV 89 4 MEKGQDHPLGALLDETGCHFCVWAPQSDKVELCLFDEQER-ELARLELPGRRGQYRFGH 62 $\mathrm{Glg} X[A$, veronii $]$

d

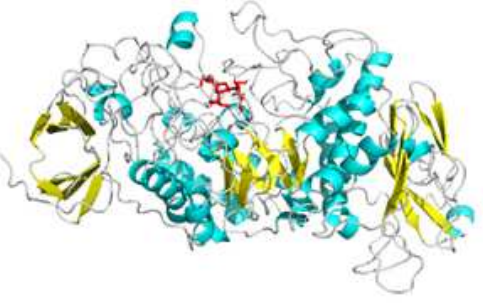

Helix Sheet Coil Cavity Fragment

GlgX [B. glumae BGR1]

249 GLTNYWGYNTIGFFAADPRYF 269

206 GLTNYWGYNPIAFFAPEPRYA 226

$\mathrm{GlgX}$ [A. veronii]

GlgX [B. glumae BGR1]

562 GNNAYCQDNEISWVDWNGID 582

521 GNN AYCQDNQISWVNWWRP 541

GlgX [A. veronii]

\section{Figure 4}

Structural presentation of sample proteins. a) Structure of P9 with alpha helixes in red. SSSH_01 $=12$, SSSH_02 $=150$. b) Structure of P6 with alpha helixes in red, SSSH_01 $=1$, SSSH_02 $=35$. c) Structure of anchor protein P5 with alpha helixes in blue and beta sheets in yellow; binding ligand1-kestose is shown in red. d) Structure of protein P19 with alpha helixes in blue and beta sheets in yellow; binding ligand1kestose is shown in red. e) Structural alignment of cavity fragments of P5 (blue) and P19 (gray) using 
align function in pymol with alignment RMSD $=0.389$; residue labels are shown in corresponding colors. f) Sample AAS alignment of P5 (GlgX [B. glumae]) and P19 (GlgX [A. veronii]) around the two cavity fragments; aligned AA of a given secondary structure or belongs to a cavity fragment is shown in the color scheme. g) Sample AAS alignment of non-cavity parts of P5 and P19, with AA of a given secondary structure shown in the color scheme.
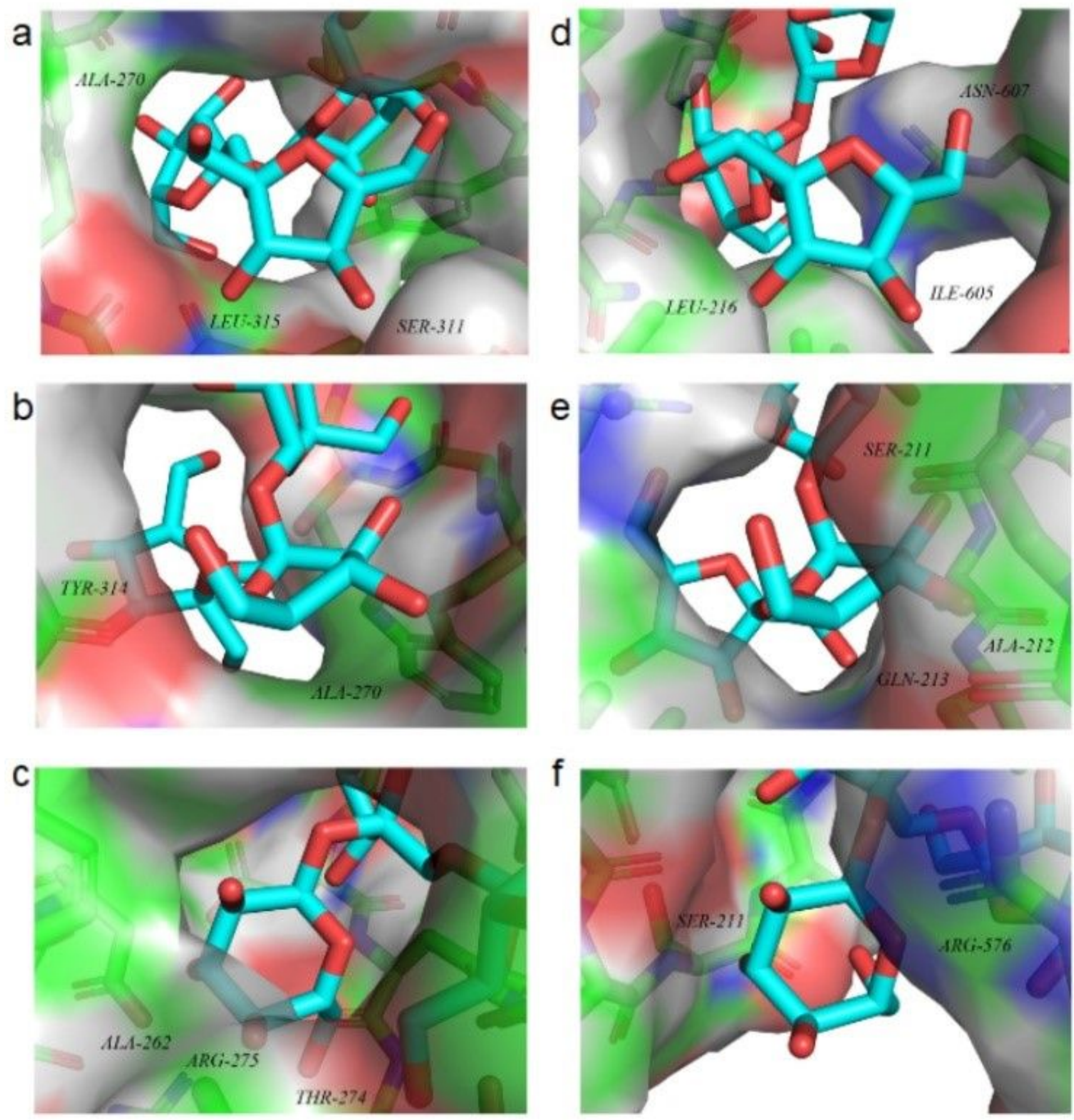

\section{Figure 5}


Predicted interaction of two CAZyme CBMs (P10 and P52) with 1-kestose. P10 and P52 are highly correlated according to group 1 features, while having no significant similarity in sequence. Similar amino acid residues are found in the radius of the interaction of both P10 and P52, including SER, ALA, ARG, and LEU. This suggests that the feature matrix may obtain inherent biological meaning. a) Interaction model of P10 viewing from beta-D-fructofuranose. b) Interaction model of P10 viewing from beta-Dfructofuranosyl residue. c) Interaction model of P10 viewing from alpha-D-glucopyranosyl residue. d) Interaction model of P52 viewing from beta-D-fructofuranose. e) Interaction model of P52 viewing frombeta-D-fructofuranosyl residue. f) Interaction model of P52 viewing from talpha-D-glucopyranosyl residue.

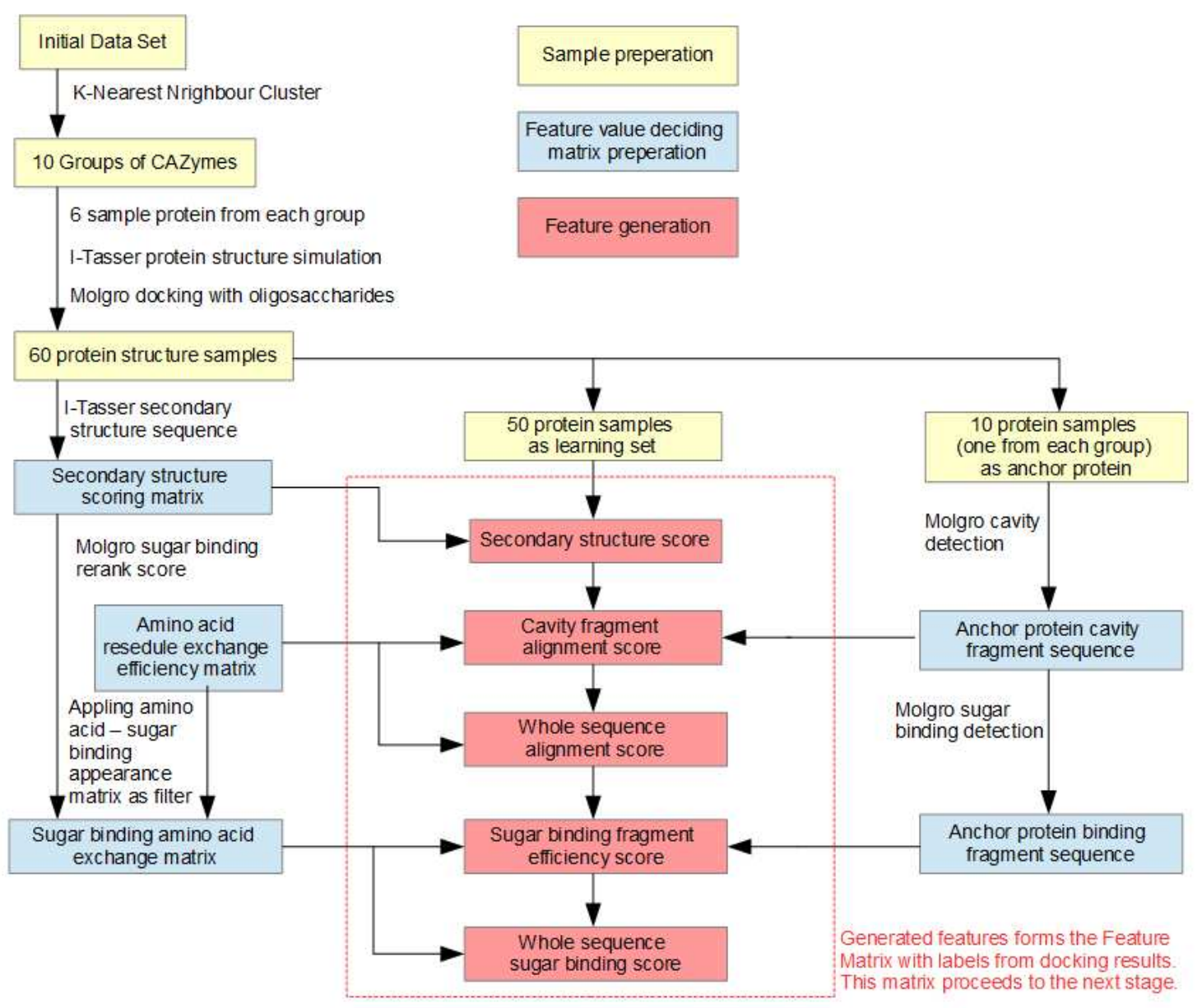

\section{Figure 6}

Flow chart for feature generation procedure. Arrows show derivation. 


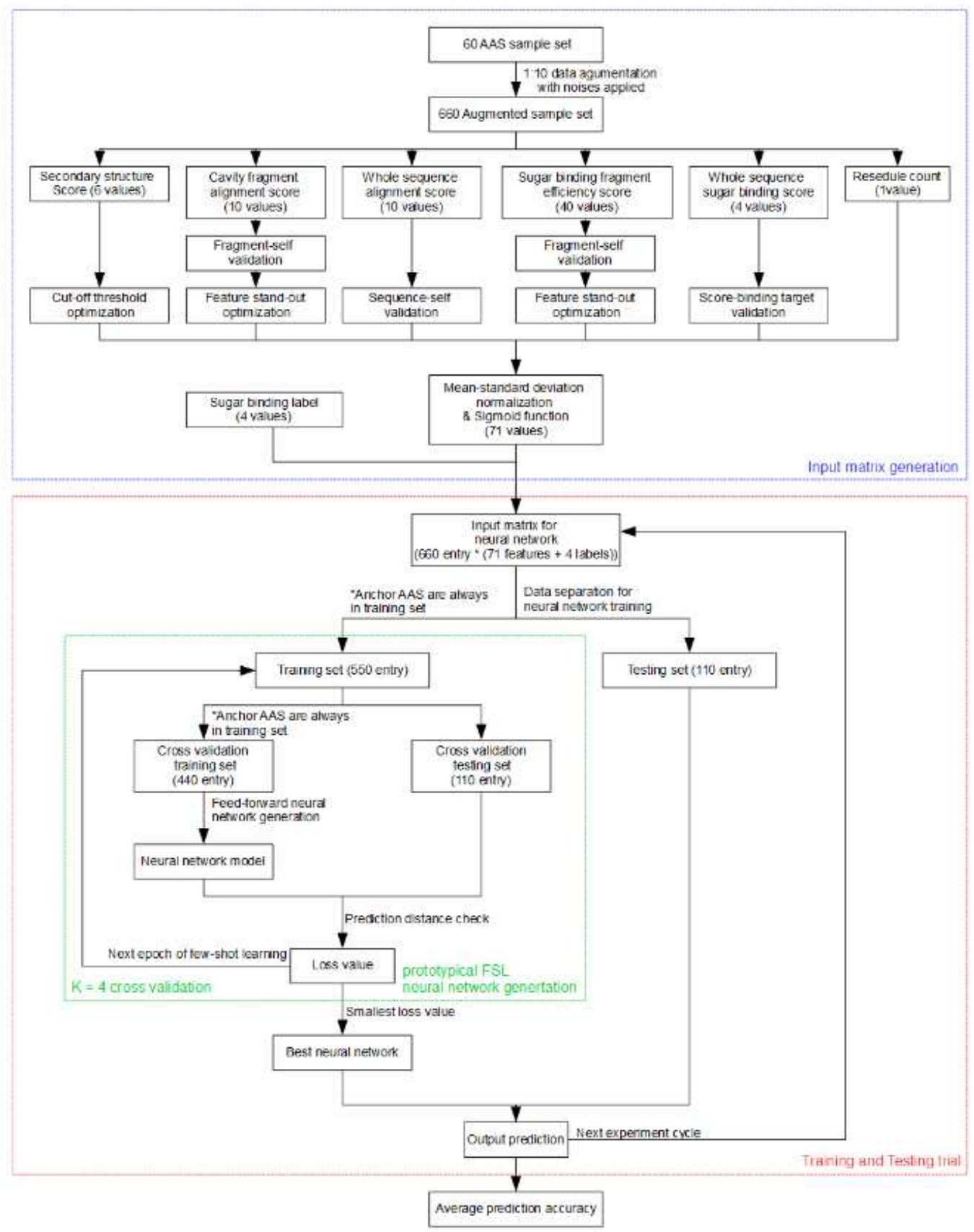

Figure 7

Flow chart of feature matrix generation and $\mathrm{pFSLNN}$ process 


\section{Algorithm 1}

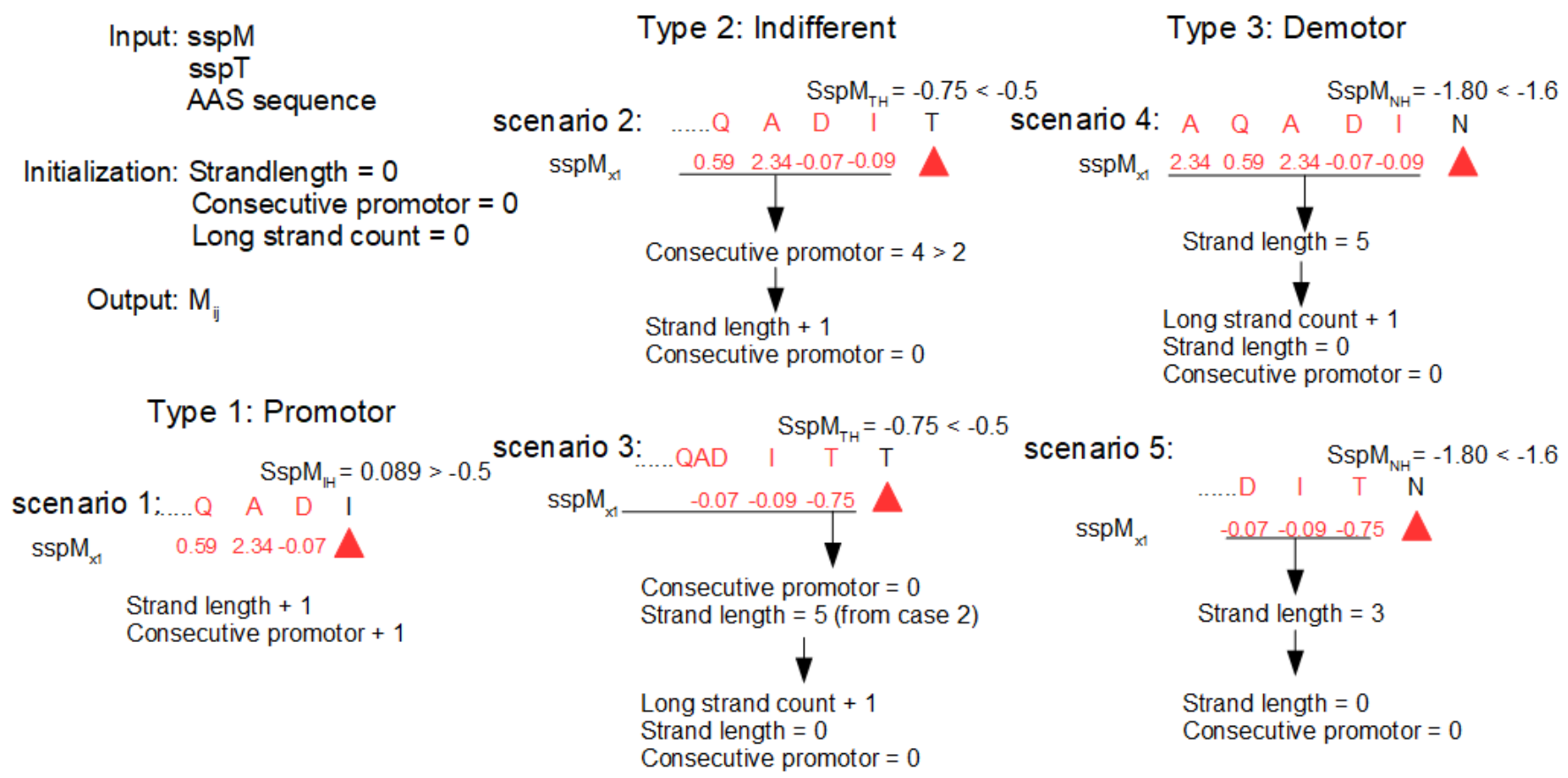

\section{Figure 8}

This figure illustrates the bases of the algorithm estimating the number of residuals of each general secondary structure type. Without loss of generality, a-Helix $(H)$ is shown as an example. A cursor scans over the input AAS to find the first $\mathrm{H}$ promoting $\mathrm{AA}$. A secondary structure strand of $\mathrm{H}$ begins. When the strand length is more than or equal to 5 , the strand is considered a long strand. As the cursor proceeds, 3 types of successor (promoting, demoting, indifferent) AA can result in 5 cases. Initial $\operatorname{sspTH}=(-0.5,-1.6$, 2).

\section{Supplementary Files}

This is a list of supplementary files associated with this preprint. Click to download.

- Supplimental1.bmp

- Supplimental2.png 